\title{
Lauroleic Acid
}

National Cancer Institute

\section{Source}

National Cancer Institute. Lauroleic Acid. NCI Thesaurus. Code C68385.

A monounsaturated medium-chain fatty acid with a 12-carbon backbone and a single double bond originating from the 1st, $2 \mathrm{nd}$, 3rd, 5th, 6th or 10 th positions from the methyl end. 